\title{
Simulation in Ultrasound Training for Obstetrics and Gynecology: A Literature Review
}

\author{
Jesica Urbina ${ }^{1}$, Stormy M Monks ${ }^{2}$, Scott B Crawford ${ }^{3}$
}

\begin{abstract}
Aim: The purpose of this review is to present the state of the art for simulation-based ultrasound training in obstetrics and gynecology. Background: Ultrasound (US) is a primary tool used in obstetrics and gynecology and has become indispensable in prenatal assessment and for diagnosing various gynecological pathologies. The core skills for US, however, are highly operator dependent. The American Institute of Ultrasound in Medicine (AIUM) assembled a multisociety task force in 2017 to develop standardized recommendations for curricula and competency assessment tools for the performance of basic OB/GYN ultrasound examinations in residency programs. Due to diminishing opportunities for training during residency programs, the task force encourages the use of US simulation during residency training and expects simulation to be a significant part of the curriculum and competency assessment process.

Methods: A literature search was conducted for the key terms "ultrasound," "OB/GYN," "simulation," and "phantoms," for works published in English from 2017-2021 with clinically relevant results reviewed.

Results: Based on the reviewed literature, there are three primary approaches for US simulation. These are grouped by their method of image generation: (1) software-based learning that occurs digitally or online through web-based programs or virtual reality (VR) systems, (2) mannequin-based, where a high-fidelity mannequin is paired with actual US images from a dataset, or 3) phantom-based training devices that simulate anatomical features for the practice of diagnostic or procedural skills. Commercially available mannequin-based simulators offer numerous pathologies for the evaluation of first and second-trimester pregnancies as well as normal anatomic features, allowing the learner to perform standard obstetric measurements and calculations in different fetal positions. Alternatively, the creation of custom US simulation trainers by a simulation center is a well-established technique to meet the needs of a specific training program. This may be to provide less expensive alternatives to commercial products, meet unique simulation requirements, or produce consumable simulation components.

Conclusion: Simulation is an important component of resident training in OB/GYN due to the diminishing opportunities during clinical rotations. There are commercially available options for training in US imaging, acquisition, interpretation, and integration into diagnostics, however, the limitations for these are that they may not be customizable and can be rigid in their learning modules and objectives. Thus, self-made models can be constructed using materials that have been characterized for their US properties. Multiple phantom materials can be aggregated to construct functional task trainers that can be used for training in the acquisition of targets along various planes. Additionally, simulators allow for training of US-guided invasive procedures, which many times are low incidence/high-risk tasks.

Clinical significance: With increasing time pressures for procedural and clinical efficiency as well as requirements for improved patient safety, clinical training opportunities are no longer a stand-alone option for resident procedural experience and training requirements. The use of simulation training in external training labs, or as a component of just-in-time training, is being used to meet these needs. Learners can gain required procedural baseline skills to allow focus on patient care delivery in the healthcare environment and less on device interaction, muscle memory training, or image interpretation.
\end{abstract}

Keywords: Gynecology, Obstetrics, Simulation, Ultrasound.

Donald School Journal of Ultrasound in Obstetrics and Gynecology (2021): 10.5005/jp-journals-10009-1816

\section{BACKGROUND}

Ultrasound (US) is a primary tool in obstetrics and gynecology and has become indispensable in prenatal assessment and for the diagnosis of various gynecological pathologies. US imaging is portable, inexpensive compared to other imaging modalities, and does not expose the patient or provider to ionizing radiation. Additionally, the real-time aspect of US imaging allows for simultaneous palpation to aid in localizing the anatomic source of pelvic pain or to examine pelvic organs. ${ }^{1}$ The core skills for US, however, are highly operator dependent and can have a high degree of subjectivity. ${ }^{1,2}$ While most providers would agree that US should be the first line of imaging for patients, it remains variable due to the lack of standardization of training and assessment. ${ }^{3}$ In response to these deficits, and given the ubiquity of US in OB/GYN, the American Institute of Ultrasound in Medicine (AIUM) assembled a multisociety task force in 2017 to develop standardized recommendations for curricula and competency assessment tools
${ }^{1-3}$ Training and Educational Center for Healthcare Simulation (TECHS), Texas Tech University Health Sciences Center, El Paso, 5001 El Paso Drive MSC-21003, El Paso, Texas, United States

Corresponding Author: Jesica Urbina, Training and Educational Center for Healthcare Simulation (TECHS), Texas Tech University Health Sciences Center El Paso, 5001 El Paso Drive MSC-21003, El Paso, Texas, United States, Phone:+1 915-215-6130, e-mail: jesica.urbina@ttuhsc.edu

How to cite this article: Urbina J, Monks SM, Crawford SB. Simulation in Ultrasound Training for Obstetrics and Gynecology: A Literature Review. Donald School J Ultrasound Obstet Gynecol 2021;15(4):359-364.

Source of support: Nil

Conflict of interest: None

for the performance of basic OB/GYN ultrasound examinations in residency programs. ${ }^{2}$

The International Society of Ultrasound in Obstetrics and Gynecology (ISUOG) has recommendations of at least 200 obstetrics

(c) The Author(s). 2021 Open Access This article is distributed under the terms of the Creative Commons Attribution 4.0 International License (https://creativecommons. org/licenses/by-nc/4.0/), which permits unrestricted use, distribution, and non-commercial reproduction in any medium, provided you give appropriate credit to the original author(s) and the source, provide a link to the Creative Commons license, and indicate if changes were made. The Creative Commons Public Domain Dedication waiver (http://creativecommons.org/publicdomain/zero/1.0/) applies to the data made available in this article, unless otherwise stated. 
scans for residents in OB/GYN, and the American College of Obstetricians and Gynecologists (ACOG) requires specific US training for at least three months or a minimum of 300 US scans as part of maternal-fetal medicine programs. ${ }^{4,5}$ Additionally, a nationwide needs assessment in Denmark concluded that two of the top five procedures in gynecological training include proficiency in US image acquisition skills or the use of an US machine. ${ }^{6}$ However, due to increasing educational demands and limited hours in residency training programs, the time dedicated to training has substantially diminished. Data from the Accreditation Council for Graduate Medical Education (ACGME) on US performance in OB/GYN residency programs indicate that the current number of US procedures performed as part of residency programs falls short of the minimum threshold required for physician qualification. ${ }^{7,8}$ Ultrasound training in residency clinical rotations with actual patients can increase cost, may increase patient discomfort, and may affect patient safety. ${ }^{9}$ Because of these concerns, access to opportunities for training can be limited. In response, the task force encourages the use of US simulation during residency training and expects simulation to be a significant part of the curriculum and competency assessment process. ${ }^{2}$

Simulation as a teaching and assessment modality is essential for procedural skill acquisition and has been shown to increase learner knowledge and skills. ${ }^{8}$ A recent (2017) review describes US simulators used in $\mathrm{OB} / \mathrm{GYN}$ and provides information on the effectiveness and validity of US simulation. ${ }^{8}$

The goal of this review is to summarize the tools for US simulation that are currently available. There are a wide variety of applications and techniques in OB/GYN that are performed at low-frequency by highly specialized operators and thus a flexible, stepwise approach to skills training has been proposed. ${ }^{9}$ Although there is a widespread use of US in OB/GYN, training in US remains a challenge. Competence is not necessarily a result of clinical experience and improvements in performance are usually attributed to sustained, deliberate practice through simulation and only rarely occur through isolated clinical practice. ${ }^{9}$ Thus, the literature supports the idea that superior performance is achieved after training using high-fidelity US simulation. ${ }^{10}$

There are four levels of skills acquisition in US training: imaging, image acquisition, interpretation of US images, and integration of findings into medical decision-making. Training in image interpretation is generally performed in real-time with volunteer or patient models, but training may also be computer-based with still and video images of US scans. Training in image acquisition is typically a hands-on process where the learner becomes familiar with the US machine to obtain and optimize a diagnostically useful image. Alternatively, training for the performance of US-guided procedures requires deliberate practice and ambidextrous handeye-probe coordination to accomplish a procedure.

A recent review suggests that simulation can be best used for the acquisition of technical skills and image optimization. ${ }^{9}$ Additionally, a learner with superior technical ability can accelerate the time it takes to achieve competence. ${ }^{9,11,12}$ Previous studies have also sought to validate the concept of using simulation for training or assessment in OB/GYN US and with rare exception, they found evidence for usefulness or validity in support of simulation as a training methodology across many medical specialties. ${ }^{9}$

This review presents current methods, tools, and techniques for training US skills in OB/GYN education. The objectives are to (1) present existing US technologies and how they relate to clinical applications, (2) describe the features of characterized ultrasoundable simulation model materials, and (3) to describe how to create US task trainers and adaptable techniques for future applications.

The current research presents convincing evidence that US simulation training leads to significant effects on learning and transferability to clinical performance and patient-perceived quality of care. Thus, US simulation training should be incorporated in every curriculum for new OB/GYN trainees. ${ }^{12,13}$

\section{Review Results}

A literature search was conducted for the key terms "ultrasound," "OB/GYN," "simulation," and "phantoms," for works published in English from 2017-2021 with clinically relevant results reviewed. A literature search was conducted for the key terms "ultrasound," "OB/GYN," "simulation," and "phantoms," for works published in English from 2017-2021 with clinically relevant results reviewed.

Based on the reviewed literature there are three primary approaches for US simulation. These are grouped by their method of image generation: (1) software-based learning that occurs digitally or online through web-based programs or virtual reality (VR) systems, (2) mannequin-based, where a high-fidelity mannequin is paired with actual US images from a dataset, or (3) phantom-based training devices that simulate anatomical features for the practice of diagnostic or procedural skills. An US phantom is a training device that can produce imaging using an actual US probe/machine and is often made from water-based and/or polymer-based materials.

Many didactic training programs exist as software, websites, or via smartphone or tablet applications, but these do not offer hands-on or experiential learning that is the hallmark of simulation-based training. Software-only US training options exist with video and guides provided by US device manufactures and from academic institutions contributing to the rapidly expanding world of free open access medical education (FOAM-ED). Many virtual simulators offer a comprehensive case-based and scenario-driven curriculum, and while there is the convenience of no requirements for specialized equipment, these offerings do not train in the techniques of image acquisition or probe manipulation, and thus they will not be discussed further in this review.

Commercially available mannequin-based simulators (SonoSim; Vimedix, CAE Healthcare; ScanTrainer, Intelligent Ultrasound; Simbionix U/S Mentor, 3DSystems) offer 3-D anatomies that are rebuilt from actual medical imaging or highly-realistic computer modeling. This approach produces realistic images based on learner action and allows the learner to maneuver a simulated US probe that senses orientation and/or position. These systems offer numerous pathologies for evaluation of first and second-trimester pregnancies as well as normal anatomic features allowing the learner to perform standard obstetric measurements and calculations in different fetal positions. ${ }^{13}$ These systems each use a unique method for identifying probe location and orientation and some have haptic feedback properties. Each can assist with demonstrating image identification but vary in the realism of image acquisition due to differences in probe tracking technique and real versus computer-created imaging.

SonoSim training products use real patient US images and will display them based on a fixed point of contact, but image display will vary based on the orientation of a special probe that senses the angle/tilt of the operator's hand using internal gyroscopic sensors. These images can be triggered based on software either from a 
set program or by reading a code from a radio-frequency (RF) tag located on a mannequin or standardized patient. ${ }^{14}$

Vimedix from CAE Healthcare ${ }^{15}$ and Simbionix U/S Mentor from 3DSystems ${ }^{16}$ both use computer-modeled images. These images are shown on a display and vary based on the position of the probe relative to an included manikin model. This allows placement and tilt of the probe to affect the acquired image and because the images are hyper-realistic, it can provide overlay and labeling of structures to help with teaching anatomy and structure identification.

The ScanTrainer, by Intelligent Ultrasound, ${ }^{17}$ also uses real patient images and adds a unique component to training by providing haptic feedback. This feedback is provided through a tripod system that is attached to a simulated US probe for a transabdominal technique or a six-axis haptic arm for transvaginal approaches. These devices allow the operator to perceive the shape of the surface and force applied to the simulated structure that is being imaged.

Phantoms can be created for specific training activities or are commercially available such as those from Blue Phantom ${ }^{18}$ (CAE Healthcare), $\mathrm{CIRS}^{19}$ and Kyoto Kagaku. ${ }^{20}$ Typically, a physical "phantom" is encased in a medium on which a non-simulated US probe is applied to produce images based on maneuvering by the operator. These devices allow for real-time scanning and practice for US-guided procedures. ${ }^{21}$

The main disadvantage of training with software or mannequin-based systems is the expense and relative lack of tactile feedback that physical phantom-based devices offer. Phantoms on the contrary, while effective at training probe manipulation and device operation, are limited by the fixed nature of the internal components and may degrade over time. Additionally, if invasive procedures are practiced, repetitive needle punctures can leave US-detectable "scarring" in the phantom and require replacement after multiple uses. ${ }^{22}$

\section{Self-made Solutions}

The creation of custom US simulation trainers by a simulation center is a well-established technique to meet the needs of specific training programs. This may be to provide less expensive alternatives to commercial products, meet unique simulation requirements, or produce consumable simulation components. More recently, the potential to produce patient-specific simulants has arisen as well. ${ }^{23}$ Commercially-produced task trainers often take a "one-size-fits-all" approach, however, customizable solutions allow for deliberate practice in high-risk, low-frequency procedures. Three-dimensional printing materials for simulation development have been studied previously and generally require combination

Table 1: Characterized materials used for US phantoms and their sonographic properties

\begin{tabular}{lcc}
\hline Product & US image appearance & Apparent depth $(\mathrm{cm})$ \\
\hline Ballistic gel $^{14}$ & Clear & $2.5-3$ \\
$\begin{array}{l}\text { Food-grade gelatin } \\
\text { Sodium polyacrylate }\end{array}$ & Clear & 3 \\
hydrogel $^{22}$ & Clear & Undefined \\
Silicone rubber $^{14}$ & Noise & \\
Polenta $^{23}$ & Noise & $4-4.5$ \\
Urethane foam $^{14}$ & Opaque & 1.5 \\
Silicone foam & Undefined \\
\hline
\end{tabular}

with other techniques, such as casting and molding to image the more dense 3-D printed forms. Other literature has examined methods to make generalizable tissue material for task trainers and measuring the fidelity of particular tissues, such as the pleura. ${ }^{24}$

The ideal material for any simulation project will, of course, depend on several factors including, but not limited to, the anatomy being simulated, imaging properties, resistance to needle passage or cutting, durability, cost, and availability. The "criterion standard" for simulation material is animal/human tissue however, it carries significant burdens including cost, limited shelf life/reusability, and limited ability to customize to a simulation task. ${ }^{25}$

\section{Phantom Materials Characterized for US Properties}

There are generally two types of US phantoms; those that mimic the acoustic properties of the tissue with regard to the speed of sound or attenuation through a given medium, and those that approximate the sonographic appearance of tissues. The physical properties of materials that can be used as phantoms and that may be useful in simulating soft tissue are listed in Table 1. The US characteristics for foams, rubbers, silicones, and gels have been previously determined from casts in standardized forms. ${ }^{15}$ In general, hydrated gels such as gelatin, ballistic gel, or sodium polyacrylate are sonolucent and give the appearance of being clear on US. Silicone rubbers tend to give the appearance of "noise" which would mimic more sonodense anatomical features, and foams, due to their trapped air content, appear opaque under US. The needle forces necessary to puncture each material were also measured and ranged from $0.05-23.34 \mathrm{~N}$. For perspective, the force necessary to puncture human skin varies from $2-5 \mathrm{~N}$, and approximately $10-20 \mathrm{~N}$ during epidural needle insertion through the ligamenta supraspinale and flavum, respectively. ${ }^{26}$ Since these materials can mimic soft tissues for imaging and interventions, there is substantial potential for the independent production of custom, high-fidelity phantoms.

Gelatin-based US phantoms have long since been implemented as simulation tools. ${ }^{21}$ Standard recommendations for the preparation of gelatin US phantoms typically use over-the-counter gelatin at lower annealing temperatures and higher concentrations than the manufacturer recommendations. ${ }^{27-29}$ This is to produce a phantom with adequate surface strength that provides tactile feedback for the trainee that is similar to tissue. Additionally, the phantom surface should have a durable surface that will not be disrupted by pressure from the US probe and is resistant to multiple needle punctures if it is used for US-guided biopsy/needle insertion training. Althoff et al. found that a latex-coated gelatin block was sufficient to obscure needle punctures and add to the durability of the phantom. ${ }^{28}$ When properly made, the echogenicity of gelatin phantoms simulate parenchymal tissue and can aid in US-guided invasive procedures when used in combination with embedded sonodense targets.

Phantoms composed of agar of varying percentages are also acceptable with regards to their fidelity to real tissue. Phantoms constructed from varying agar concentrations with and without additives have been characterized for their US properties such as image quality and durability and compared to gelatin and human tissue. For example, a five percent by-mass agar model containing small amounts of suspended wheat flour is a phantom that could generate an US image that remarkably resembles real tissue. ${ }^{30}$ The advantages over gelatin phantoms are that the agar does not require refrigeration, is resistant to biofouling and desiccation, mimics parenchymal tissue, can withstand high-volume training, 
and can be recycled to make new phantoms. The addition of wheat flour or corn starch is, however, labor-intensive to achieve homogeneity. An interesting observation was that even if the added flour clumped or was irregularly dispersed in the medium, the resulting phantom mimicked the irregularity of real tissue. Abdominal wall fat, for example, has a heterogeneous echo pattern, and phantoms with unevenly dispersed starch or flour additives are heterogeneous in echogenicity. ${ }^{19}$

The addition of psyllium, a hydrophilic mucilloid fiber, has also been used to make clear gels opaque and to achieve the unique US scatter patterns that may be used to simulate ovarian parenchyma. ${ }^{18,31}$ The primary advantage of psyllium over starch or flour is that when the mixture is prepared, further mixing is not necessary to achieve an even suspension in the scattering medium.

Ballistic gel has also been characterized for use as an US phantom. It is similar in echogenicity to agar and gelatin, however, it has the added benefit of having a much longer shelf-life and does not tear as easily. ${ }^{32}$ Ballistic gel that has been impregnated with psyllium has also been characterized and was found to create tissue-like densities. Additionally, molding techniques can be used to create lumens that can retain fluid, self-seal, and can be aspirated to demonstrate evidence of needle placement. This is especially useful for US-guided invasive procedures, or when approximating amniotic fluid volume. It can be used to make US-training models quickly and with only a fraction of the cost when compared to commercially available options. ${ }^{26}$

Recently, precooked polenta has also been explored as an US phantom. Originally designed as a tool for mimicking soft tissue infections, this model can be used to aid in the imaging of features such as a blighted ovum or adnexal masses to include corpus luteum cysts, dermoid cysts, ectopic pregnancy, etc., however, the image quality is limited beyond a depth of $1.5 \mathrm{~cm} .{ }^{33}$ Minimal preparation was required for this model where gelatin-encased paintballs were embedded in polenta and characterized with US. The resulting model closely replicated superficial soft tissue abscesses. Additionally, the polenta can be reshaped easily and the phantom punctured or incised multiple times.

An additional self-made food-based training phantom for US skills related to OB/GYN training involved the use of tofu as a substrate that approximates myometrium on US imaging. This phantom is described as having gummy bears embedded within a block of tofu and covered with US gel in a small hollow to mimic early pregnancy. ${ }^{22}$ This training device was termed the "tofuterus" by its creator. ${ }^{34}$ Tapioca starch pearls with small amounts of pudding in plastic bags that are secured in the folds of nylon leggings near a central block of tofu allowed for concomitant visualization of "ovaries" and "cysts" when the entire product was submerged in a water bath.

As US becomes the standard of care in OB/GYN, there is an increasing demand to develop better and more cost-effective simulation models to educate trainees. These self-made phantom solutions offer a viable option for training in imaging, identification, and US-guided procedures.

\section{Effectiveness of Simulation for US Training}

All of the studies reviewed reported that there was an improvement in US skills acquisition or competence after simulation-based training. One recent study reported that simulation-based US training is as effective as training with real patients and is especially beneficial for novice trainees. ${ }^{35}$ Rosen et al. conducted a prospective, randomized, single-blinded trial with obstetric trainees who had minimal previous US exposure. ${ }^{35}$ In this study, learner assessment for scans in mid-trimester fetal brain anatomy was chosen as a surrogate for fetal anatomy US training. All participants were given a didactic session regarding the required planes, underwent a pretest, and then proceeded to train with either an US simulator or a "real" patient volunteer. The results found that pretest competence was similar in both groups. Additionally, both groups had significant score improvement following training regardless of whether the learners trained on a simulated (mean score pretest 15.9 vs post-test $28.9 ; p<0.05$ ) or real patient (mean score pretest 13.3 vs post-test $24.6 ; p<0.04$ ).

Additionally, a recent review that evaluated the learning and assessment of US skills in OB/GYN for eight studies found that trainees and experienced clinicians struggle with the technical aspects of US image optimization. ${ }^{36}$ The authors found that the use of simulation-based US training on the technical aspects of the US instruments led to improvements in all subsequent aspects of performance in a clinical setting, including a decrease in patient discomfort and their perceived safety and confidence in their US operator.

Another study found that general practice (GP) residents who underwent simulation-based US training produced sonographic images that were of improved quality versus those residents who received clinical training alone. ${ }^{37}$ The GP residents participated in a one-day simulation-based US training session. Assessment of the impact of the training on TVS (transvaginal ultrasound scan) image quality was conducted with a group of GP residents who attended the simulation training course and were compared to scans generated by GP residents who did not attend the course and had only clinical experience in US. The authors found that US image quality for sonograms that were collected two months after the training were significantly better in the group that received the US simulation-based training when compared to those collected from practitioners who had only clinical training. ${ }^{37}$

\section{Discussion}

Characterized materials for phantom construction can aid the healthcare simulation technology specialist in forming an entire simulated system. The characteristics of individual phantoms can be used to simulate anatomical features when mixing materials and allow for target features to be clearly distinguished from the surrounding medium. One such example is training in amniocentesis for fetal lung maturity testing or genetic evaluation for aneuploidy. While it is no longer a required skill for OB/GYN residency, there are still some general OB/GYNs who need to perform amniocentesis. There are commercially available task trainers, however, the static models are limited in their ability to train users for the variability that is encountered in clinical settings. Thus, self-made solutions are a viable option for complex US-guided procedures. For example, a task trainer for amniocentesis procedures can be made from ballistics gel or gelatin with embedded sodium polyacrylate hydrogel "water beads" that are sonolucent to obscure sonodense targets, and placed over a manikin to simulate a gravid abdomen. ${ }^{38,39}$ This model allows learners to have deliberate practice in the stereotactic skills required to maneuver a needle under US guidance and to hit a specific target during amniocentesis.

The AIUM recommends that all providers performing US-guided invasive procedures be competent in a core set of skills, including in-plane and out-of-plane needle guidance. To allow for the practice and assessment of these core skills, a novel task trainer was developed by Nitsche et al. ${ }^{40}$ that allows the user to practice 
hand-eye coordination with general needle guidance skills. The authors obtained validity evidence in the content and response process domains according to the Standards for Educational and Psychological Testing. Historically, each invasive procedure has been taught independently of US-guided needle procedures, however, the stepwise approach to training does not allow for enough time to teach/learn more complex procedures during a residency term. Thus, the task trainer allows a learner to perfect key elements involved in invasive tasks such as planning the approach, selection of the instrument insertion site, localization of the needle, and using US for needle guidance to the target without losing US visualization. ${ }^{40}$

Although there are differences in how to perform US-guided invasive procedures, the core skills learned from deliberate practice using a task trainer transfer to procedures such as amniocentesis, chorionic villus sampling (CVS), and percutaneous umbilical blood sampling. ${ }^{26}$

Studies have shown that even novice practitioners can achieve competence in the performance of OB/GYN US procedures when being trained by simulation alone. ${ }^{41,42}$ These conclusions were based on studies that compare simulation-based curriculum with conventional didactic teaching of US and practice.

\section{Conclusion}

Simulation is an important component of resident training in $\mathrm{OB} / \mathrm{G} Y N$ due to the diminishing opportunities during clinical rotations. There are commercially available options for training in US imaging, acquisition, interpretation, and integration into diagnostics, however, the limitations for these are that they may not be customizable in their learning modules and objectives. Thus, self-made models can be constructed using materials that have been characterized for their US properties. Multiple phantom materials can be aggregated to construct functional task trainers that can be used for US training in the acquisition of targets along various planes as well as training for US-guided invasive procedures, which many times are low-incidence, high-risk tasks.

\section{Clinical Significance}

With increasing time pressures for procedural and clinical efficiency as well as requirements for improved patient safety, clinical training opportunities are no longer a stand-alone option for resident procedural experience and training requirements. Recent studies suggest that consideration should be given to the integration of simulation training for the acquisition of US core skills. There has been a steady decline in clinical training opportunities for procedures such as amniocentesis and CVS since the advent of cell-free fetal DNA tests from maternal serum. Thus, alternative training methods are essential. ${ }^{43}$ The use of US simulation in the field of OB/GYN has the potential to replace many of the lost opportunities for real-life training and allow trainees to gain core US skills. The value of simulation has been established for many procedures and skills before moving to clinical practice. ${ }^{23}$ Learners can gain required procedural baseline skills to allow focus on patient care delivery in the healthcare environment and less on device interaction, muscle memory training, or image interpretation.

\section{Acknowledgments}

The authors would like to thank the Texas Tech University Health Sciences Center El Paso Office of Faculty Development for their guidance and support during the development of this manuscript.

\section{References}

1. Abuhamad A, Minton KK, Benson $C B$, et al. Obstetric and gynecologic ultrasound curriculum and competency assessment in residency training programs: consensus report. American J Obstet Gynecol 2018;218(1):29-67. DOI: 10.1002/jum.14519

2. Tolsgaard MG. Assessment and learning of ultrasound skills in Obstetrics \& Gynecology [dissertation]. University of Copenhagen, Faculty of Health and Medical Sciences; 2017.

3. Benacerraf BR, Minton KK, Benson CB, et al. Proceedings: Beyond Ultrasound First Forum on improving the quality of ultrasound imaging in obstetrics and gynecology. American J Obstet Gynecol 2018;218(1): 19-28. DOI: 10.1016/j.ajog.2017.06.033

4. ISUOG: update on proposed minimum standards for ultrasound training for residents in Ob/Gyn Ultrasound Obstet Gynecol 1996;8: 363-366. DOI: 10.1046/j.1469-0705.1996.08050363.x

5. American Board of Obstetrics and Gynecology 2021 [Accessed 14 June 2021] http://www.abog.org/downloads.asp.

6. Nayahangan LJ, Konge L, Møller-Skuldbøl IM, et al. A nationwide needs assessment to identify and prioritize technical procedures for simulation in obstetrics and gynaecology: a Delphi study. J Obstet Gynaecol Can 2020;42(4):409-419. DOI: 10.1016/j. jogc.2019.08.043

7. Accreditation Council for Graduate Medical Education (ACGME). Obstetrics and gynecology case logs: national data report. Available at: https:// www.acgme.org/Portals/0/PFAssets/ProgramResources/ OBGYNCaseLogInfo.pdf?ver=2021-05-03-161144-103. pdf. Accessed June 22, 2021.

8. American Institute of Ultrasound in Medicine. AlUM ultrasound practice accreditation. Available at: http://www.aium.org/ accreditation/ accreditation.aspx. Accessed June 22, 2021.

9. Arya S, Dwivedi A, Mulla ZD, et al. Effectiveness of ultrasound simulation in obstetrics and gynecology education: a state-of-the-Art review. Donald Sch JUltrasound Obstet Gynecol 2017;11:115-125. DOI: 10.5005/jp-journals-10009-1512

10. Dromey BP, Peebles DM, Stoyanov DV. A systematic review and meta-analysis of the use of high-Fidelity simulation in obstetric ultrasound. Simul Healthc 2021;16(1):52. DOI: 10.1097/SIH.0000000000000485

11. Arya S, Mulla ZD, Kupesic Plavsic S. Role of pelvic ultrasound simulation. Clin Teach 2018;15(6):457-461. DOI: 10.1111/tct.12714

12. Taksøe-Vester C, Dyre L, Schroll J, et al. Simulation-based ultrasound training in obstetrics and gynecology: a systematic review and meta-analysis. Ultraschall Med 2020. DOI: 10.1055/a-1300-1680

13. Mahesh RMD, Menon A, Sherif L, et al. Evaluation of the Role of Simulation Training in Nuchal Translucency Measurement. Int J Contemp Med Surg Radiol 2019;4(2):B47-B50. DOI: 10.21276/ijcmsr.2019.4.2.11

14. The easiest way to learn ultrasonography ${ }^{\oplus}[$ Internet]. SonoSim. [cited 2021 Jun 01]. Available from: http://www.sonosim.com/

15. Simulation based Medical Education Solutions: Cae Healthcare [Internet]. CAE Healthcare - Medical Simulation Training Solutions. [cited 2021 Jun 01]. Available from: https://www.caehealthcare.com/.

16. Simulators [Internet]. Simbionix. 2020 [cited 2021 Jun 01]. Available from: https://simbionix.com/.

17. OBGYN [Internet]. Intelligent Ultrasound. 2021 [cited 2021 Jun 01]. Available from: https://www.intelligentultrasound.com/

18. See how blue phantom improves patient safety [Internet]. Blue Phantom Ultrasound Training Medical Models and Ultrasound Simulators. [cited 2021 Jun 01]. Available from: https://www. bluephantom.com/

19. Fetal Ultrasound Biometrics Phantom [Internet]. CIRS. [cited 2021 Jun 01]. Available from: https://www.cirsinc.com/product-category/ ultrasound/

20. Fetus Ultrasound Examination Phantom "Space fan-st" [Internet]. Fetus Ultrasound Examination Phantom "SPACE FAN-ST" | KYOTO KAGAKU. [cited 2021 Jun 01]. Available from: https://www. kyotokagaku.com/en/products_data/us-7_en/ 
21. Collins MK, Chuang M, Deering S, et al. Basic gynecologic encounters and procedures. Comprehensive Healthcare Simulation: Obstetrics and Gynecology 2019;pp.171-176. Springer, Cham. DOI: 10.1007/978-3-319-98995-2_16

22. Crawford SB, Baily LW, Monks SM. Comprehensive Healthcare Simulation: Operations, Technology, and Innovative practice 2019. Springer International Publishing. ISBN-13: 978-3030153779; ISBN-10: 3030153770

23. Caldwell J, Mooney JJ. Analysis of soft tissue materials for simulation development. Simul Healthc 2019;14(5):312-317. DOI: $10.1097 / \mathrm{SIH} .0000000000000382$

24. Mooney JJ, Sarwani N, Coleman ML, et al. Evaluation of 3D printed materials for simulation by computed tomography and ultrasound imaging. Simul Healthc 2017;12(3):182-188. DOI: $10.1097 / \mathrm{SIH} .0000000000000217$

25. Sultan SF, Shorten G, lohom G. Simulators for training in ultrasound guided procedures. Med Ultrason 2013;15(2):125-131. DOI: $10.11152 / \mathrm{mu} .2013 .2066 .152 . s f s 1 \mathrm{gs} 2$

26. Esterer B, Hollensteiner M, Schrempf A, et al. Characterization of tissue properties in epidural needle insertion on human specimen and synthetic materials. J Mech Behav Biomed Mater 2020;110:103946. DOI: 10.1016/j.jmbbm.2020.103946

27. Bude RO, Adler RS. An easily made, low-cost, tissue-like ultrasound phantom material.J Clin Ultrasound 1995;23(4):271-273. DOI: 10.1002/jcu.1870230413

28. Althoff S, Sudhir A, Woods WA. A study of the surface characteristics of homemade ultrasound phantoms. Crit Ultrasound J 2010;2(2): 53- 57. DOI: 10.1007/s13089-010-0042-3

29. Gerstenmaier JF, McCarthy CJ, Brophy DP, et al. Evaluation of the particulate concentration in a gelatin-based phantom for sonographically guided lesion biopsy. J Ultrasound Med 2013;32(8): 1471-1475. DOI: 10.7863/ultra.32.8.1471

30. Earle M, De Portu G, DeVos E. Agar ultrasound phantoms for low-cost training without refrigeration. Afr J Emerg Med 2016;6(1):18-23. DOI: 10.1016/j.afjem.2015.09.003

31. Elshamy T, Jayaprakasan K. Sonographic assessment of polycystic ovaries. Gynaecological Ultrasound Scanning. 2020;87-99. DOI: $10.1017 / 9781108149877.007$

32. Amini R, Kartchner JZ, Stolz LA, et al. A novel and inexpensive ballistic gel phantom for ultrasound training. World J Emerg Med 2015;6(3): 225. DOI: 10.5847/wjem.j.1920-8642.2015.03.012
33. Augenstein JA, Yoshida H, Lo MD, et al. A readily available, inexpensive, and reusable simulation model for teaching ultrasound-guided abscess identification and drainage. J Emerg Med 2016;50(3): 462-465. DOI: 10.1016/j.jemermed.2015.12.020

34. O'Reilly M, Hoffmann S, Hess L, et al.. Low-tech, high-fidelity models for a robust ultrasound curriculum. Council on Resident Education in Obstetrics and Gynecology (CREOG) and the Association of Professors of Gynecology and Obstetrics (APGO) Annual Meeting. Orlando 2017.

35. Rosen $H$, Windrim R, Lee $Y M$, et al. Simulator based obstetric ultrasound training: a prospective, randomized single-blinded study. J Obstet Gynaecol Can 2017;39(3):166-173. DOI: 10.1016/j. jogc.2016.10.009

36. Tolsgaard MG. A multiple-perspective approach for the assessment and learning of ultrasound skills. Perspect Med Educ 2018;7(3): 211-213. DOI: 10.1007/s40037-018-0419-8

37. Le Lous M, De Chanaud N, Bourret A, et al. Improving the quality of transvaginal ultrasound scan by simulation training for general practice residents. Adv Simul 2017;2(1):1-5. DOI: 10.1186/s41077-017-0056-z

38. Oropeza N, Radtke S. Amniocentesis simulator. Southwest Region Simulation Educator and Operations Conference at Texas Tech University Health Sciences Center El Paso. Poster presentation 2020.

39. Maher JE, Kleinman GE, Lile W, et al. The construction and utility of an amniocentesis trainer. Am J Obstet Gynecol 1998;179(5):1225-1227. DOI: 10.1016/s0002-9378(98)70136-x

40. Nitsche JF, Shumard KM, Brost BC. Development and assessment of a novel task trainer and targeting tasks for ultrasound-guided invasive procedures. Acad Radiol 2017;24(6):700-708. DOI: 10.1016/j. acra.2016.10.008

41. Maul H, Scharf A, Baier P, et al. Ultrasound simulators: experience with the SonoTrainer and comparative review of other training systems. Ultrasound Obstet Gynecol 2004;24(5):581-585. DOI: $10.1002 /$ uog. 1119

42. Chalouhi GE, Bernardi V, Gueneuc A, et al. Evaluation of trainees' ability to perform obstetrical ultrasound using simulation: challenges and opportunities. Am J Obstet Gynecol 2016;214(4): 525.e1-525.e8. DOI: 10.1016/j.ajog.2015.10.932

43. Nitsche JF, Brost BC. Obstetric Ultrasound-Guided Invasive Procedure Simulation. Comprehensive Healthcare Simulation: Obstetrics and Gynecology 2019;pp.157-168. Springer, Cham. DOI: 10.1007/978-3-319-98995-2_15 